\title{
Partial Differential Equations
}

\section{On a new class of functions related to VMO}

\author{
Haïm Brezis ${ }^{\mathrm{a}}$, Hoai-Minh Nguyen ${ }^{\mathrm{b}}$ \\ a Rutgers University, Dept. of Math., Hill Center, Busch Campus, 110 Frelinghuysen Road, Piscataway, NJ 08854, USA \\ b Courant Institute, New York University, 251 Mercer St., New York, NY 10012, USA
}

\section{A R T I C L E I N F O}

\section{Article history:}

Received and accepted 23 November 2010

Available online 3 January 2011

Presented by Haïm Brezis

\section{A B S T R A C T}

In this Note, we compare the space VMO and the spaces

$$
\mathbf{I}_{p}:=\left\{g \in L^{1}(\Omega ; \mathbb{R}) ; \quad \int_{\substack{\Omega \\|g(x)-g(y)|>\delta}} \frac{1}{|x-y|^{d+p}} \mathrm{~d} x \mathrm{~d} y<+\infty \quad \forall \delta>0\right\}
$$

where $\Omega$ is a bounded open subset of $\mathbb{R}^{d}, d \geqslant 1$, and $p \geqslant 0$. In particular, we prove that $\mathbf{I}_{d} \subset V M O$. This sharpens the well-known result stating that $W^{s, p} \subset V M O$ for $0<s<1$ and $s p=d$. Moreover, we establish that $V M O$ is much bigger than $\mathbf{I}_{d}$ by showing that $V M O \not \subset \mathbf{I}_{1}$. We also present some results when the double integral above is taken on the set $\{(x, y) \in$ $\left.\Omega \times \Omega ;\left|e^{i g(x)}-e^{i g(y)}\right|>\delta\right\}$.

(C) 2010 Académie des sciences. Published by Elsevier Masson SAS. All rights reserved.

R É S U M É

Dans cette Note, nous comparons l'espace VMO et les espaces

$$
\mathbf{I}_{p}:=\left\{g \in L^{1}(\Omega ; \mathbb{R}) ; \quad \int_{\substack{\Omega \\|g(x)-g(y)|>\delta}} \frac{1}{|x-y|^{d+p}} \mathrm{~d} x \mathrm{~d} y<+\infty \quad \forall \delta>0\right\},
$$

où $\Omega$ est un ouvert borné de $\mathbb{R}^{d}, d \geqslant 1$, et $p \geqslant 0$. En particulier, nous prouvons que $\mathbf{I}_{d} \subset$ $V M O$. Ceci améliore le résultat bien connu affirmant que $W^{s, p} \subset V M O$ pour $0<s<1$ et $s p=d$. D'autre part, nous prouvons que $V M O$ est plus grand que $\mathbf{I}_{d}$; en fait $V M O \not \subset \mathbf{I}_{1}$. Nous présentons aussi des résultats lorsque l'intégrale double ci-dessus est prise sur l'ensemble $\left\{(x, y) \in \Omega \times \Omega ;\left|e^{i g(x)}-e^{i g(y)}\right|>\delta\right\}$.

(C) 2010 Académie des sciences. Published by Elsevier Masson SAS. All rights reserved.

\section{Main results}

The principal motivation of this note comes from the study of the topological degree of maps from the sphere $\mathbb{S}^{d}$ into itself. It was proved in [2] that the degree is well-defined for maps $u \in V M O\left(\mathbb{S}^{d}, \mathbb{S}^{d}\right)$. In fact it suffices to assume that

$$
\limsup _{|Q| \rightarrow 0} f_{Q}\left|u(x)-f_{Q} u(y) \mathrm{d} y\right| \mathrm{d} x<1
$$

E-mail addresses: brezis@math.rutgers.edu (H. Brezis), hoaiminh@cims.nyu.edu (H.-M. Nguyen). 
and the constant 1 is optimal. Note that (1.1) is satisfied in particular if

$$
\limsup _{|Q| \rightarrow 0} f_{Q} f|u(x)-u(y)| \mathrm{d} x \mathrm{~d} y<1 / 2 \text {. }
$$

On the other hand, it was proved in [6] that the degree of $u$ is well-defined when

$$
\iint_{\substack{\mathbb{S}^{d} \mathbb{S}^{d} \\ u(x)-u(y) \mid>\delta}} \frac{1}{|x-y|^{2 d}} \mathrm{~d} x \mathrm{~d} y<+\infty \text { for some } \delta \in\left(0, \ell_{d}\right),
$$

where $\ell_{d}=\sqrt{2+2 /(d+1)}$; and moreover

$$
|\operatorname{deg} u| \leqslant C_{d} \underset{\substack{\mathbb{S}^{d} \mathbb{S}^{d} \\|u(x)-u(y)| \geqslant \ell_{d}}}{ } \frac{1}{|x-y|^{2 d}} \mathrm{~d} x \mathrm{~d} y .
$$

Therefore it is natural to investigate the possible connection between the spaces $V M O, B M O$, and the class of functions satisfying conditions of the type (1.2). We introduce the following definitions. Let $\Omega$ be a smooth bounded domain in $\mathbb{R}^{d}$, and $0 \leqslant p<+\infty$. Set

$$
\mathbf{I}_{p}=\left\{g \in L^{1}(\Omega ; \mathbb{R}) ; \quad \iint_{\substack{\Omega \\|g(x)-g(y)|>\delta}} \frac{1}{|x-y|^{d+p}} \mathrm{~d} x \mathrm{~d} y<+\infty \forall \delta>0\right\}
$$

and

$$
\mathbf{J}_{p}=\left\{g \in L^{1}(\Omega ; \mathbb{R}) ; \quad \iint_{\substack{\Omega \\|g(x)-g(y)|>\delta}} \frac{1}{|x-y|^{d+p}} \mathrm{~d} x \mathrm{~d} y<+\infty \text { for some } \delta>0\right\} .
$$

The case $p<0$ is not interesting because $\mathbf{I}_{p}=\mathbf{J}_{p}$ coincides with $L^{1}(\Omega)$.

Here is a brief list of properties:

A) $\mathbf{I}_{p}$ and $\mathbf{J}_{p}$ are vector spaces.

B) $\mathbf{I}_{p} \subset \mathbf{I}_{q}$ and $\mathbf{J}_{p} \subset \mathbf{J}_{q}$ if $p \geqslant q$.

C) $C(\bar{\Omega}) \subset \mathbf{I}_{p} \subset \mathbf{J}_{p}$ for all $p \geqslant 0$.

D) $W^{s, p} \subset \mathbf{I}_{s p}$ for all $s \in(0,1)$ and $p>1$.

We recall here that, for $0<s<1$ and $p>1$,

$$
W^{s, p}(\Omega):=\left\{g \in L^{p}(\Omega) ;|g|_{W^{s, p}<+\infty}\right\},
$$

where

$$
|g|_{W^{s, p}}^{p}:=\iint_{\Omega} \frac{|g(x)-g(y)|^{p}}{|x-y|^{d+s p}} \mathrm{~d} x \mathrm{~d} y .
$$

E) $W^{1, p} \subset \mathbf{I}_{p}$ for all $p>1$. More precisely (see [5]), for $p>1$ and $g \in W^{1, p}(\Omega)$, we have

$$
\delta^{p} \quad \iint_{\substack{\Omega \\|g(x)-g(y)|>\delta}} \frac{1}{|x-y|^{d+p}} \mathrm{~d} x \mathrm{~d} y \leqslant C_{d, p, \Omega} \int_{\Omega}|\nabla g|^{p} \mathrm{~d} x .
$$

The constant $C_{d, p, \Omega}$ blows up as $p \rightarrow 1$ and in fact $W^{1,1} \not \subset \mathbf{I}_{1}$ (an example due to A. Ponce is presented in [5]).

F) $\mathbf{J}_{p} \subset L^{p^{*}}$ with $1<p<d$ and $\frac{1}{p^{*}}=\frac{1}{p}-\frac{1}{d}$ (see [7]). This is an extension of the classical Sobolev embedding $W^{1, p} \subset L^{p^{*}}$. It is not true that $\mathbf{I}_{d} \subset L^{\infty}$ (clearly $W^{s, p} \subset \mathbf{I}_{d}$ and it is known that $W^{s, p} \not \subset L^{\infty}$ for $s p=d$ ). Even when $p>d$, it is not true that $\mathbf{I}_{p} \subset L^{\infty}$ (see [7]); this is in contrast with the Morrey-Sobolev embedding.

It is known that $W^{s, p} \subset V M O$ for all $d \geqslant 1,0<s \leqslant 1$, and $s p=d$; see e.g. [2]. In view of D), one may wonder whether the larger space $\mathbf{I}_{d}$ is also contained in $V M O$. The answer is positive: 
Theorem 1. Let $d \geqslant 1$. Then

a) $\mathbf{J}_{d} \subset B M O$.

b) $\mathbf{I}_{d} \subset V M O$.

Remark 1. The exponent $d$ in Theorem 1 is optimal in the following sense: if $d \geqslant 1$ and $0 \leqslant p<d$ then $\mathbf{I}_{p} \not \subset B M O$. Indeed, let $q>1$ and $0<s<1$ be such that $p<s q<d$. Then

$$
W^{s, q} \subset \mathbf{I}_{s q} \subset \mathbf{I}_{p} \quad \text { and } \quad W^{s, q} \not \subset B M O .
$$

This implies $\mathbf{I}_{p} \not \subset B M O$.

The proof of Theorem 1 is essentially based on the following proposition which is proved in [7]. In what follows, we denote by $Q$ the unit cube in $\mathbb{R}^{d}$.

Proposition 1. Let $d \geqslant 1, p \geqslant 1, \delta>0$, and $g \in L^{1}(Q)$. Then

$$
\iint_{Q}|g(x)-g(y)|^{p} \mathrm{~d} x \mathrm{~d} y \leqslant C_{d, p}\left[\int_{\substack{Q \\|g(x)-g(y)|>\delta}} \frac{\delta^{p}}{|x-y|^{d+p}} \mathrm{~d} x \mathrm{~d} y+\delta^{p}\right],
$$

for some positive constant $C_{d, p}$ depending only on $d$ and $p$.

Remark 2. The proof of Proposition 1 is quite delicate and it would be desirable to find a more elementary argument, even for $d=1$. It makes use of ideas introduced in Bourgain-Nguyen [1]. It also relies on the John-Nirenberg inequality [4]. Some inequalities related to (1.4) and their applications in the theory of Sobolev spaces are presented in [7].

One may ask whether the inclusions in Theorem 1 are strict. It turns out that VMO is "much bigger" than $\mathbf{I}_{d}$. In fact, we have a stronger assertion:

Theorem 2. Let $d \geqslant 1$. Then there exists $g \in V M O$ such that $g \in W^{s, p}$ for all $s \in(0,1), p>1$ with $s p<1$, and $g \notin \mathbf{J}_{1}$, i.e.,

$$
\iint_{\substack{Q \\|g(x)-g(y)|>\delta}} \frac{1}{|x-y|^{d+1}} \mathrm{~d} x \mathrm{~d} y=+\infty, \quad \forall \delta>0 .
$$

Remark 3. Let $0 \leqslant t<1$ and $d \geqslant 1$. We have not been able to construct a function $g \in V M O$ such that $g \notin \mathbf{J}_{t}$. It might be true, for example, that $V M O \subset \mathbf{J}_{0}$; this is an open problem.

We next present a variant of Proposition 1.

Theorem 3. Let $1 \leqslant p<+\infty$ and $0<\delta<\sqrt{3}$. We have, for all $g \in C(\bar{Q}, \mathbb{R})$.

$$
\iint_{Q}|g(x)-g(y)|^{p} \mathrm{~d} x \mathrm{~d} y \leqslant C_{d, p, \delta}\left(\int_{\substack{Q \\\left|e^{i g(x)}-e^{i g(y)}\right|>\delta}} \frac{1}{|x-y|^{d+p}} \mathrm{~d} x \mathrm{~d} y+1\right) .
$$

Moreover, the restriction that $\delta<\sqrt{3}$ is optimal.

Theorem 3 has been proved in [3] when $p=1$ and $d=1$. Already in this case the proof is quite elaborate. The case $d=1$ and $p>1$ can be proved using exactly the same argument as in the case $d=1$ and $p=1$. The proof in the case $d>1$ is a consequence of the 1- $d$ case using the argument in Step 2 of the proof of [7, Theorem 1].

Theorem 3 fails if we delete the assumption that $g \in C(\bar{Q})$. In fact, for each $n \in \mathbb{N}_{+}$, take $g_{n}(x)=0$ on $(0,1 / 2) \times(0,1)^{N-1}$ and $g_{n}(x)=2 \pi n$ for $x \in(1 / 2,1) \times(0,1)^{N-1}$. Then

$$
\iint_{Q}\left|g_{n}(x)-g_{n}(y)\right|^{p} \mathrm{~d} x \mathrm{~d} y \rightarrow \infty \text { as } n \rightarrow \infty,
$$


and

$$
\iint_{\substack{Q \\\left|e^{i g_{n}(x)}-e^{i g_{n}(y)}\right|>\delta}} \frac{1}{|x-y|^{d+p}} \mathrm{~d} x \mathrm{~d} y=0, \quad \forall \delta>0 .
$$

Theorem 3 implies Proposition 1 when $g \in C(\bar{Q})$. However we do not know how to deduce Proposition 1 from Theorem 3 for a general function $g \in L^{1}(Q)$ because we are not able to pass to the limit in the RHS of (1.4) when $g$ is regularized.

Another natural question is whether (1.5) holds for $g \in V M O(Q)$. We know that the answer is positive if $d=1$ and $p=1$ (see [3]). By the same method as in [3], one can prove that the answer holds for $d=1$ and $p>1$.

We also have

Theorem 4. Let $d \geqslant 1$ and $k \in \mathbb{N}_{+}$be such that $1 \leqslant k \leqslant d$. Then there exists $g \in V M O(Q)$ such that $g \in W^{s, p}(Q)$ for all $s \in(0,1)$, $p>1$, and $s p<k$, and

$$
\iint_{\substack{Q \\ Q}} \frac{\mathrm{d} x \mathrm{~d} y}{|x-y|^{d+k}}=+\infty, \quad \forall 0<\delta<2 .
$$

Detailed proofs of these results will be presented elsewhere.

\section{Acknowledgement}

The first author is partially supported by NSF Grant DMS-0802958.

\section{References}

[1] J. Bourgain, H.-M. Nguyen, A new characterization of Sobolev spaces, C. R. Acad. Sci. Paris, Ser. I 343 (2006) 75-80.

[2] H. Brezis, L. Nirenberg, Degree theory and BMO, Part I: Compact manifolds without boundaries, Selecta Math. 1 (1995) 197-263.

[3] H. Brezis, H.-M. Nguyen, On the distributional Jacobian of maps from $\mathbb{S}^{N}$ into $\mathbb{S}^{N}$ in fractional Sobolev and Hölder spaces, Ann. of Math., available online: 31 December 2009.

[4] F. John, L. Nirenberg, On functions of bounded mean oscillation, Comm. Pure Appl. Math. 14 (1961) 415-426.

[5] H.-M. Nguyen, Some new characterizations of Sobolev spaces, J. Funct. Anal. 237 (2006) 689-720.

[6] H.-M. Nguyen, Optimal constant in a new estimate for the degree, J. d'Analyse Math. 101 (2007) 367-395.

[7] H.-M. Nguyen, Some inequalities related to Sobolev norms, Calc. Var. Partial Differential Equations, published online: 12 October 2010, doi:10.1007/ s00526-010-0373-8. 